\title{
Ce que les grammaires françaises omettent de signaler
}

\author{
Darja Damić Bohač \\ Faculté de Philosophie et Lettres \\ ddbohac@ffzg.hr
}

Dans cet article nous nous proposons de présenter quelques points de grammaire que les grammaires françaises écrites par les locuteurs natifs, et surtout les manuels de FLE, omettent de signaler. Et même lorsque ces points de grammaire y sont mentionnés, les descriptions simplifiées et les explications sommaires ne permettent pas de déjouer les pièges d'apprentissage auxquels un public allophone doit faire face.

Mots clés : approche contextualisée, analyse contrastive, grammaire, manuels de FLE, difficultés, CECR

\section{Introduction}

Précisons tout d'abord que notre intention n'était pas de bouleverser la tradition scolaire de l'enseignement du français. Toutefois nous avons voulu éclairer certains faits de langue ou plutôt les présenter dans une nouvelle optique adaptée au public visé dans le but de faciliter l'apprentissage/acquisition du français - dans notre contexte ce sont les étudiants croatophones en philologie française, dont pour la majorité le français n'est qu'une deuxième langue étrangère, ce qui devrait impliquer aussi la prise en compte des interférences interlinguales outre que celles de leur langue maternelle. ${ }^{1}$

Nous formons l'espoir qu'une telle description contextualisée pourrait profiter même aux locuteurs natifs francophones, car elle permet d'expliquer les phénomènes de langue sous un autre angle qui prend en considération non seulement les remarques contrastives, mais aussi les remarques intralinguales, voire diachroniques là où cela s'avère nécessaire.

Le cadre de ce travail nous a obligé de nous limiter à quelques difficultés sélectionnées à titre d'exemple qui semblent à première vue mineures, mais qui

1 Pour plus de détail sur le déroulement des études de langue et littérature françaises au sein de la Faculté de Philosophie et Lettres de Zagreb, notamment sur les contenus de formation en compétences linguistiques et communicatives voir Berlengi/Damić (2014 : 115-125). 
persistent même à des niveaux élevés de maîtrise. De même nous n'avons pas pu aborder une approche contextualisée ${ }^{2}$ ni présenter les lacunes des grammaires descriptives, normatives, scolaires et autres, et surtout celles des manuels de FLE.

\section{Le nombre d'éléments nécessaires pour former le superlatif français}

Commençons par la comparaison adjectivale, plus précisément par le superlatif relatif dont le point le plus important, et peut-être le plus négligé par les grammaires, les manuels et les professeurs de français s'adressant à un public croatophone, est le nombre d'éléments nécessaires pour avoir un superlatif relatif en français : deux éléments suffisent dans une comparaison à former un superlatif français (la plus belle des deux sœurs, le meilleur des deux frères), alors qu'un superlatif croate en exige trois (naljepša od triju sestara, najbolji od trojice braće).

Evidemment, ceci ne pose aucun problème à des locuteurs natifs s'ils sont monolingues! Ces derniers ne risquent pas de tomber dans le piège des interférences.

\section{La pronominalisation des compléments d'objet indirects datifs et non datifs}

Une des difficultés majeures est certainement la pronominalisation des compléments d'objet directs, partitifs, indirects datifs ou non datifs qui recèle de nombreux écueils suivant le degré d'actualisation du noyau nominal objet, la forme affirmative ou négative de la phrase, la catégorie sémantique animé / non animé du référent, la forme simple ou composée du verbe et, surtout, suivant les propriétés rectionnelles du verbe qui gouvernent la forme directe ou indirecte de son complément ainsi que le choix de la préposition.

C'est la raison pour laquelle nous redoutons les conséquences des tendances de simplification introduites dans l'analyse grammaticale des programmes scolaires français qui réduisent le groupe verbal au simple prédicat sans que soient identifiés les différents types de compléments indirects datifs et non datifs.

Nous devons ici nous limiter seulement à la pronominalisation des compléments d'objet indirects datifs, où se neutralise l'opposition animé / non animé (Je le lui préfère correspond à Je préfêre Pierre à Jacques et à Je préfère le café au thé. Ceci est passé sous silence par les grammaires, à la différence de la pronominalisation des compléments d'objet indirects non datifs où s'opposent les référents animés et non animé : Je pense à lui et J'y pense correspondant à Je pense à Pierre / Je pense à notre enfance.

Nous convenons que pour un locuteur natif français, la pronominalisation conjointe (atone) des compléments datifs s'opposant à la pronominalisation

2 Voir Damić Bohač (2019) qui présente une ébauche de grammaire contextualisée condensant les difficultés auxquelles se heurtent les étudiants croatophones et propose des descriptions plus opératoires pour essayer de remédier aux analogies intra et interlinguales. 
disjointe (tonique) des compléments d'objet indirects non datifs ne devrait pas poser de problème, bien que confronté à la pronominalisation d'un exemple anodin comme Le jour succède à la nuit, il aurait des hésitations à employer la forme conjointe lui. De leur côté les apprenants de français vont peiner à distinguer deux types de compléments en $\grave{a} \mathbf{N}$ qui répondent à la même question (à qui ?), mais qui ont un comportement syntaxique différent lequel se manifeste de manière formelle uniquement dans la pronominalisation :

Tamara ressemble à son frère. / Tamara pense à son frère.

Tamara ressemble à qui ? / Tamara pense à qui ?

Tamara lui ressemble. / Tamara pense à lui.

Les grammaires omettent de préciser que le verbe répondre régit deux constructions, l'une dative, l'autre indirecte NON dative :

Tamara a répondu à Martin $\rightarrow$ Tamara lui ai répondu.

Tamara a répondu à sa lettre $\rightarrow$ Tamara y a répondu.

D'ailleurs son équivalent croate odgovoriti régit, lui aussi, deux constructions indirecte, l'une au datif, l'autre à l'accusatif prépositionnel :

Tamara je odgovorila Martinu $\rightarrow$ Tamara mu je odgovorila.

Tamara je odgovorila na njegovo pismo $\rightarrow$ Tamara je na to odgovorila.

Une fois que la pronominalisation est maîtrisée, il faut bouleverser les acquis en signalant les cas où la pronominalisation des compléments datifs doit se faire par les formes disjointes (toniques). La raison en est l'incompatibilité des formes conjointes datives lui et leur, du pronom réfléchi se et des formes accusatives (me, te, nous, vous et se). ${ }^{3}$

\section{Les constructions verbales qui ont disparu de l'usage}

Alors que les grammaires françaises mentionnent volontiers que les verbes désobéir, obéir et pardonner ont changé de construction au cours des siècles, qu'ils sont devenus transitifs indirects à complément datif, mais qu'ils gardent toujours la trace de leur ancienne transitivité directe dans la transformation passive (Elle a été désobéie / obéie / pardonnée), elles tiennent caché le fait que les verbes apprendre

3 Heureusement que les grammaires françaises, dont V. Riegel / Pellat / Rioul (1999 : 201) signalent cette difficulté : Je me confie à lui / à elle. Je te confie à lui / à elle. Il se confiait à moi. Elle nous a recommandés à lui. L'occasion s'offre à lui. Je me joins à toi. Et non *Je me lui confie. *Je te lui confie. ${ }^{*}$ Il se me confiait. *Elle nous lui a recommandés. ${ }^{*}$ 'occasion se lui offre. *Je me te joins. 
et enseigner régissaient autrefois deux accusatifs (ou compléments directs) $\mathrm{d}^{\prime}$ origine latine et qu'on disait aux $\mathrm{XVI}^{\mathrm{e}}$ et $\mathrm{XVII}{ }^{\mathrm{e}}$ siècles enseigner les enfants la grammaire (v. Guiraud 1974 : 61). Aujourd'hui, cette construction doublement accusative est évidemment une erreur qui refait surface là où on s'y attendrait le moins : dans les premières versions des mémoires de Master (filière didactique), car nos étudiants font le transfert de la construction à double accusatif croate $u c ̌ i t i$ koga što au lieu de faire suivre les verbes apprendre et enseigner d'un complément d'objet direct (qqch) et d'un complément datif (⿳亠口冖 qqn).

\section{Le passif}

La transformation passive est présentée en détail dans les grammaires et manuels français sans que soit cependant précisée sa fonction communicationnelle - la thématisation du rhème rendue par l'antéposition de l'objet de l'action dont la transformation passive n'est qu'un des moyens.

Pierre bat Paul $\rightarrow$ Paul est battu par Pierre.

En croate, la transformation passive est beaucoup moins fréquente, car le système casuel diminue l'importance de l'ordre des mots et l'objet peut être facilement antéposé Pavla tuče Petar. ${ }^{4}$ Cette mobilité de l'objet en croate est la raison pour laquelle dans les productions orales et écrites de nos étudiants il n'est pas rare de trouver un complément d'objet direct à la place réservée au sujet sans que cette dislocation de l'objet soit reprise par le pronom personnel correspondant (Paul, Pierre l'a battu). ${ }^{5}$

\section{La périphrase factitive}

La maîtrise de la périphrase factitive donne bien du fil à retordre aux croatophones dont la langue maternelle ne connaît pas ce type de construction (v. Damić Bohač 1996, 2016, 2019 : 173-182). Les grammaires françaises donnent des explications détaillées sur les semi-auxiliaires factitifs faire et laisser ou les verbes de perception qui se font suivre de l'infinitif, de même que sur les différentes possibilités de construction (directe ou indirecte) de l'agent. Pourtant elles ne mentionnent pas que dans certains contextes l'emploi de la périphrase factitive n'est pas le résultat du choix entre le sens implicitement factitif (Mon voisin construit une maison) et explicitement factitif (Mon voisin a fait construire une maison par / à un entrepreneur.), mais que la périphrase factitive s'y impose (avec se faire) :

Pierre s'est fait opérer des amygdales. ${ }^{6}$

4 Rares sont les cas en croate où le nominatif a la même forme que l'accusatif, comme dans Hajduk je pobijedio Dinamo.

5 V. Damić Bohač 2016 : 357, 2019 : 153-164.

6 Le passif y est possible aussi : Il a été opéré des amygdales. 
Dans cet exemple le sens ne peut pas être implicitement factitif comme c'est le cas en croate (Petar je operirao krajnike) et la phrase *Pierre a opéré, les amygdales est agrammaticale. Même un spécialiste, en chirurgie cervico-faciale n'aurait pas eu l'idée d'effectuer cette opération sur lui-même, mais sur des patients : Pierre est fatigué d'avoir opéré des amygdales toute la matinée.

Les grammaires $n$ 'insistent pas sur le fait que la construction factitive permet aussi d'introduire un agent supplémentaire nécessaire à la coréférence des sujets :

Sa réussite lui a tourné la tête au point de lui faire oublier ses origines modestes. (Et non : Sa réussite lui a tourné la tête au point de lui *oublier ses origines modestes. L'équivalent croate est Uspjeh mu je zavrtio glavom tako da je zaboravio na svoje skromno porijeklo.).

De même, le sens causatif de la périphrase n'est guère mis en avant. Celui-ci est trop souvent spontanément rendu par un complément circonstanciel de cause (comme c'est le cas en croate Koljena su mu se savijala pod težinom / zbog težine sanduka ce qui donnerait en français : Ses genoux pliaient sous le poids du coffre / à cause du poids du coffre. Et ce n'est qu'après avoir été sollicités, que les étudiants se souviennent de la construction factitive de sens causatif où son sujet est la cause directe de l'action: Le poids du coffre lui faisait plier les genoux.

Dans les reformulations, il est plus probable que nos étudiants aient spontanément recours à la transformation passive (Il a été renvoyé par la direction.) qu'à la construction factitive (Il s'est fait renvoyer par la direction.).

Les grammaires ne précisent pas qu'une même périphrase factitive (par exemple faire manger) peut, selon le contexte, avoir des sens différents. Le degré de responsabilité du sujet du verbe faire n'est pas signalé, le caractère volontaire ou non, avantageux ou non encore moins.

Il faut faire manger le bébé. (cr. Treba nahraniti bebu.)

Tu ne me feras pas manger cela! (cr. Nećeš me natjerati da to pojedem.)

Le prisonnier s'est fait manger par les cannibales. (cr. Kanibali su pojeli zarobljenika.)

Ainsi, dans Le prisonnier s'est fait manger par les cannibales, l'agent de la construction factitive correspond au sujet de la construction active en croate Kanibali su pojeli zarobljenika. Le sujet de la construction factitive française, sans avoir exercé sa volonté sur l'agent faisant l'action exprimée par l'infinitif, est en fait la cause de l'action qui est due à un mauvais concours de circonstances.

La construction faire marcher peut aussi véhiculer plusieurs sens : faire marcher un appareil (cr. uključiti aparat), faire marcher un invalide (cr. postići da prohoda), faire marcher quelqu'un (cr. nasamariti koga).

\section{Conclusion}

L'enseignement de la grammaire d'une langue étrangère au niveau universitaire devrait être approfondi et complété par les difficultés spécifiques à la langue maternelle des apprenants. Une telle approche contextualisée accompagnée, là où c'est nécessaire, par des remarques contrastives rendrait

7 Voir Beacco / Kalmbach / Suso López (2014 : 3-17) et Beacco (2017). 
l'enseignement /apprentissage de la langue plus efficace. ${ }^{7}$ En attendant qu'une grammaire conçue et écrite, ou simplement complétée par des contributions d'auteurs allophones voie le jour, nous sommes témoins d'une baisse de niveau considérable des compétences linguistiques de nos étudiants inscrits en première année de Licence.

La progression grammaticale prévue dans les méthodes communicatives et actionnelles utilisées dans les établissements du secondaire est inadéquate, car elle ne tient pas compte des difficultés. Ceci a pour conséquence des connaissances lacunaires et approximatives, une production à syntaxe rudimentaire favorisant la fossilisation. Une redéfinition de la progression est nécessaire non seulement au niveau des méthodes mais aussi au niveau du Cadre européen commun de référence pour les langues et des documents qui l'accompagnent, car le fait de négliger la composante grammaticale en subordonnant la langue en tant que système à la langue en tant que moyen de communication a fait ses victimes. Nous nous en rendons compte à chaque rentrée.

\section{Références bibliographiques}

Beacco, Jean-Claude / Kalmbach, Jean-Michel / Suso López, Javier (2014). Les contextualisations de la description du français dans les grammaires étrangères : Présentation, Langue française, 2014/1 n 181, pp. 3-17.

Beacco, Jean-Claude (2017). Les activités grammaticales : réflexivité, progressivité, contextualité, in Francontraste III : Structuration, langage, discours et au-delà [éd. Pavelin Lešić, B.], CIPA, Mons, pp. 35-44.

Berlengi, Vedrana / Damić Bohač, Darja (2017). Strani jezik kao predmet poučavanja na neofilološkim studijima - na primjeru studija francuskog jezika i književnosti, in Jezik kao predmet proučavanja i jezik kao predmet poučavanja, Zbornik radova Hrvatskog društva za primijenjenu lingvistiku [éd. Diana Stolac, Anastazija Vlastelić], Zagreb : Srednja Europa, HDPL, pp. 45-55.

Damić Bohač, Darja (1996). Faire + l'infinitif, une approche contrastive, Studia Romanica et Anglica Zagrabiensia, vol. XLI [éd. Karlo Budor], Zagreb : Facultas Philosophica Univesitatis Studiorum Zagrabiensis, pp. 201-210.

Damić Bohač, Darja (2016). Kako olakšati usvajanje i učenje stranog jezika - na primjeru studenata francuskog jezika , in Metodologija i primjena lingvističkih istraživanja, Zbornik radova Hrvatskog društva za primijenjenu lingvistiku [éd. Udier, Sanda Lucija, Cergol Kovačević, Kristina], Zagreb : Srednja Europa, HDPL. pp. 349-362.

Damić Bohač, Darja (2017). Complémentation verbale, nominale et adjectivale en français et en croate in Francontraste III : Structuration, langage, discours et au-delà, Tome 2 : Sciences du langage [éd. Bogdanka Pavelin Lešić], Mons : CIPA. pp. 123-134.

Damić Bohač, Darja (2018). Le retour à la grammaire dans l'enseignement des langues étrangères, in Poglavlja iz romanske fillogije : u čast profesora $\mathrm{Au-}$ 
gusta Kovačeca o njegovu 80. rođendanu, Zagreb: FFpress, Filozofski fakultet Sveučilišta u Zagrebu,. pp. 205-217.

Damić Bohač, Darja (2019). Difficultés de la langue française vues par les croatophones, Zagreb : FFpress, Faculté de Philosophie et Lettres, Université de Zagreb / Filozofski fakultet Sveučilišta u Zagrebu.

Grevisse, Maurice (1969). Le Bon Usage, 9e édition, Editions Duculot, S.A. Gembloux

Guiraud, Pierre (1974). La syntaxe du français, Paris : Presses Universitaires de France

Riegel, Martin / Pellat, Jean-Christophe / Rioul, René (1999). Grammaire méthodique du français, Presses Universitaires de France, Paris.

\section{Ono što francuske gramatike ne spominju}

U ovome radu želimo prikazati kontekstualizirani pristup nastavi francuskog jezika kakav nudimo na studiju jezika i književnosti kroz opise jezičnih pojava kojima francuske gramatike i udžbenici francuskog jezika, čiji su autori izvorni govornici, ne pridaju značaj ili ih ne uočavaju. Nadamo se da ćemo takvim pristupom kompenzirati manjkavosti modernih metoda koje imaju za posljedicu zapostavljanja gramatike stranih jezika u srednjim školama, ali i ujedno dati drugačiji pogled na funkcioniranje francuskog jezika.

Ključne riječi: kontekstualizirani pristup, kontrastivna analiza, gramatika, udžbenici francukog kao stranog jezika, poteškoće, ZEROJ 
